\title{
Certification of Point-of-Care Ultrasound Competency
}

\author{
Nilam J. Soni, MD, MS ${ }^{1,2 *}$, David M. Tierney, MD³, Trevor P. Jensen, MD, MS ${ }^{4}$, Brian P. Lucas, MD, MS 5,6
}

\begin{abstract}
${ }^{1}$ Division of General \& Hospital Medicine, The University of Texas School of Medicine at San Antonio, San Antonio, Texas; ${ }^{2}$ Section of Hospital Medicine, South Texas Veterans Health Care System, San Antonio, Texas; ${ }^{3}$ Abbott Northwestern Hospital, Department of Medical Education, Minneapolis, Minnesota; ${ }^{4}$ Division of Hospital Medicine, Department of Medicine, University of California San Francisco, San Francisco, California; ${ }^{5}$ Medicine Service, White River Junction VA Medical Center, White River Junction, Vermont; ${ }^{6}$ Geisel School of Medicine at Dartmouth College, Hanover, New Hampshire.
\end{abstract}

Any conversation about point-of-care ultrasound (POCUS) inevitably brings up discussion about credentialing, privileging, and certification. While credentialing and privileging are institution-specific processes, competency certification can be extramural through a national board or intramural through an institutional process.

Currently, no broadly accepted national board certification for POCUS exists; however, some specialty boards, such as emergency medicine, already include competency in POCUS. Thus, many institutions grant POCUS privileges to emergency medicine physicians based solely on their national board certification. In contrast, most hospitalists are certified by the American Board of Internal Medicine, which does not include competency in POCUS. Some hospitalists have pursued extramural certificate programs offered by professional organizations, such as the American College of Chest Physicians. The currently available extramural certificate programs can certify basic competency in POCUS knowledge and skills. But none of them can deem a provider competent in POCUS, which requires mastery of knowledge, image acquisition, image interpretation, and clinical integration (Figure). Image acquisition and interpretation skills are learned at varying rates. Those skills, followed by an understanding of how to integrate POCUS findings into clinical care of patients, are ones that cannot be acquired after a weekend training course. ${ }^{1}$

Some institutions have begun to develop intramural certification pathways for POCUS competency in order to grant privileges to hospitalists. In this edition of the Journal of Hospital Medicine, Mathews and Zwank ${ }^{2}$ describe a multidisciplinary collaboration to provide POCUS training, intramural certification, and quality assurance for hospitalists at one hospital in Minnesota. This model serves as a real-world example of how institutions are addressing the need to certify hospitalists in basic POCUS competency. After engaging stakeholders from radiology, critical care, emergency medicine, and cardiology, institutional standards were developed and hospitalists were assessed for basic POCUS competency. Certification included

\footnotetext{
*Address for correspondence and reprint requests: Nilam J. Soni, MD, MS, 7703 Floyd Curl Drive, MC 7982, San Antonio, TX 78229; Telephone: 210-7436030; Fax: 210-358-0647; E-mail: sonin@uthscsa.edu
}

Received: March 23, 2017; Revised: May 12, 2017; Accepted: May 19, 2017 2017 Society of Hospital Medicine DOI 10.12788/jhm.2812

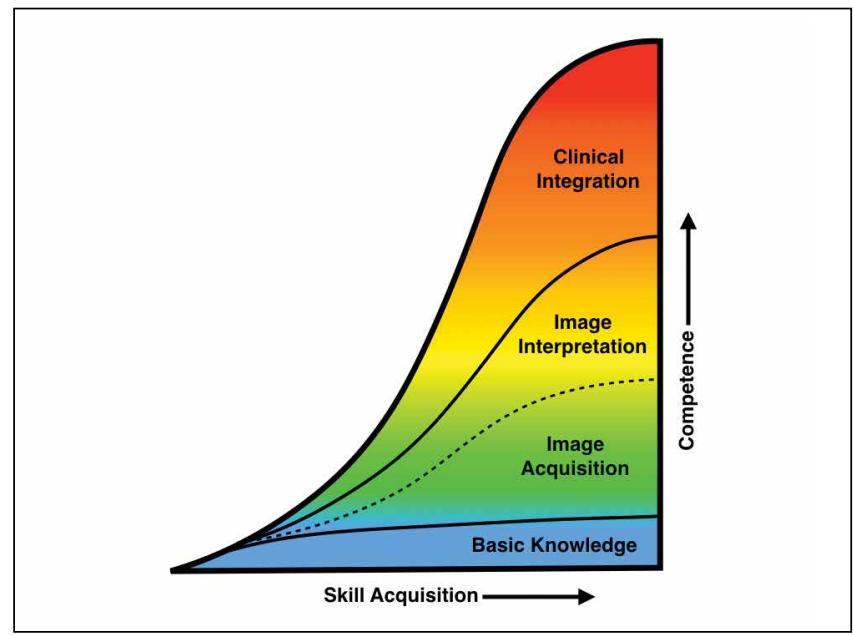

FIG. Competency in point-of-care ultrasound requires mastery of different skills. After gaining basic knowledge of ultrasonography, image acquisition and interpretation skills can be mastered. Clinical integration of ultrasound findings requires baseline competence in clinical medicine.

assessments of hospitalists' knowledge, image acquisition, and image interpretation skills. The model described by Mathews did not assess competency in clinical integration but laid the groundwork for future evaluation of clinical outcomes in the cohort of certified hospitalists.

Although experts may not agree on all aspects of competency in POCUS, most will agree with the basic principles outlined by Mathews and Zwank. Initial certification should be based on training and an initial assessment of competency. Components of training should include ultrasound didactics, mentored hands-on practice, independent hands-on practice, and image interpretation practice. Ongoing certification should be based on quality assurance incorporated with an ongoing assessment of skills. Additionally, most experts will agree that competency can be recognized, and formative and summative assessments that combine a gestalt of provider skills with quantitative scoring systems using checklists are likely the best approach.

The real question is, what is the goal of certification of POCUS competency? Development of an institutional certification process demands substantive resources of the institution and time of the providers. Institutions would have to invest in equipment and staff to operate a full-time certification program, given the large number of providers that 
use POCUS and justify why substantive resources are being dedicated to certify POCUS skills and not others. Providers may be dissuaded from using POCUS if certification requirements are burdensome, which has potential negative consequences, such as reverting back to performing bedside procedures without ultrasound guidance or referring all patients to interventional radiology.

Conceptually, one may speculate that certification is required for providers to bill for POCUS exams, but certification is not required to bill, although institutions may require certification before granting privileges to use POCUS. However, based on the emergency medicine experience, a specialty that has been using POCUS for more than 20 years, billing may not be the main driver of POCUS use. A recent review of 2012 Medicare data revealed that $<1 \%$ of emergency medicine providers received reimbursement for limited ultrasound exams. ${ }^{3}$ Despite the Accreditation Council for Graduate Medical Education (ACGME) requirement for POCUS competency of all graduating emergency medicine residents since 2001 and the increasing POCUS use reported by emergency medicine physicians, 4,5 most emergency medicine physicians are not billing for POCUS exams. Maybe use of POCUS as a "quick look" or extension of the physical examination is more common than previously thought. Although billing for POCUS exams can generate some clinical revenue, the benefits for the healthcare system by expediting care, ${ }^{6,7}$ reducing ancillary testing, ${ }^{8,9}$ and reducing procedural complications ${ }^{10,11}$ likely outweigh the small gains from billing for limited ultrasound exams. As healthcare payment models evolve to reward healthcare systems that achieve good outcomes rather than services rendered, certification for the sole purpose of billing may become obsolete. Furthermore, concerns about billing increasing medical liability from using POCUS are likely overstated because few lawsuits have resulted from missed diagnoses by POCUS, and most lawsuits have been from failure to perform a POCUS exam in a timely manner. ${ }^{12,13}$

Many medical students graduating today have had some training in POCUS ${ }^{14}$ and, as this new generation of physicians enters the workforce, they will likely view POCUS as part of their routine bedside evaluation of patients. If $\mathrm{PO}$ CUS training is integrated into medical school and residency curricula, and national board certification incorporates basic POCUS competency, then most institutions may no longer feel obligated to certify POCUS competency locally, and institutional certification programs, such as the one described by Mathews and Zwank, would become obsolete.

For now, until all providers enter the workforce with basic competency in POCUS and medical culture accepts that ul- trasound is a diagnostic tool available to any trained provider, hospitalists may need to provide proof of their competence through intramural or extramural certification. The work of Mathews and Zwank provides an example of how local certification processes can be established. In a future edition of the Journal of Hospital Medicine, the Society of Hospital Medicine Point-of-Care Ultrasound Task Force will present a position statement with recommendations for certification of competency in bedside ultrasound-guided procedures.

Disclosure: Nilam Soni receives support from the U.S. Department of Veterans Affairs, Quality Enhancement Research Initiative (QUERI) Partnered Evaluation Initiative Grant (HX002263-01A1). Brian P. Lucas receives support from the Department of Veterans Affairs, Veterans Health Administration, Office of Research and Development and Dartmouth SYNERGY, National Institutes of Health, National Center for Translational Science (UL1TR001086). The contents of this publication do not represent the views of the U.S. Department of Veterans Affairs or the United States Government.

\section{References}

1. Bahner DP, Hughes D, Royall NA. I-AIM: a novel model for teaching and performing focused sonography. J Ultrasound Med. 2012;31:295-300.

2. Mathews BK, Zwank M. Hospital Medicine Point of Care Ultrasound Credentialing: An Example Protocol. J Hosp Med. 2017;12(9):767-772.

3. Hall MK, Hall J, Gross CP, et al. Use of Point-of-Care Ultrasound in the Emergency Department: Insights From the 2012 Medicare National Payment Data Set. J Ultrasound Med. 2016;35:2467-2474.

4. Amini R, Wyman MT, Hernandez NC, Guisto JA, Adhikari S. Use of Emergency Ultrasound in Arizona Community Emergency Departments. J Ultrasound Med. 2017;36(5):913-921.

5. Herbst MK, Camargo CA, Jr., Perez A, Moore CL. Use of Point-of-Care Ultrasound in Connecticut Emergency Departments. J Emerg Med. 2015;48:191-196.

6. Kory PD, Pellecchia CM, Shiloh AL, Mayo PH, DiBello C, Koenig S. Accuracy of ultrasonography performed by critical care physicians for the diagnosis of DVT. Chest. 2011;139:538-542.

7. Lucas BP, Candotti C, Margeta B, et al. Hand-carried echocardiography by hospitalists: a randomized trial. Am J Med. 2011;124:766-774

8. Oks M, Cleven KL, Cardenas-Garcia J, et al. The effect of point-of-care ultrasonography on imaging studies in the medical ICU: a comparative study. Chest. 2014;146:1574-1577.

9. Koenig S, Chandra S, Alaverdian A, Dibello C, Mayo PH, Narasimhan M. Ultrasound assessment of pulmonary embolism in patients receiving CT pulmonary angiography. Chest. 2014;145:818-823.

10. Mercaldi CJ, Lanes SF. Ultrasound guidance decreases complications and improves the cost of care among patients undergoing thoracentesis and paracentesis. Chest. 2013;143:532-538.

11. Patel PA, Ernst FR, Gunnarsson CL. Ultrasonography guidance reduces complications and costs associated with thoracentesis procedures. J Clin Ultrasound. 2012;40:135-141.

12. Stolz L, O'Brien KM, Miller ML, Winters-Brown ND, Blaivas M, Adhikari S. A review of lawsuits related to point-of-care emergency ultrasound applications. West J Emerg Med. 2015;16:1-4.

13. Blaivas M, Pawl R. Analysis of lawsuits filed against emergency physicians for point-of-care emergency ultrasound examination performance and interpretation over a 20-year period. Am J Emerg Med. 2012;30:338-341.

14. Bahner DP, Goldman E, Way D, Royall NA, Liu YT. The state of ultrasound education in U.S. medical schools: results of a national survey. Acad Med. 2014;89:1681-1686. 